\title{
Neuer Therapie-Ansatz bremst die Entzündung in den Gelenken
}

Mitarbeiter des Zentrums für Pathophysiologie, Infektiologie und Immunologie der MedUni Wien haben eine Makrophagen-Untergruppe charakterisiert, welche stark entzündungshemmend wirken kann. In Kombination mit dem Antirheumatikum Methotrexat könnte sich daraus künftig eine neue Therapie-Option ergeben.

\begin{abstract}
_ Die im Journal „Frontiers in Immunology" veröffentlichte Studie [1] von Dr. Anna Ohradanova-Repic et al. zeigt, dass nicht nur entzündungsfördernde Makrophagen (M1) in Gelenken mit rheumatoider Arthritis vorhanden sind, sondern auch eine neue Population von Makrophagen, die bei entsprechender Förderung die Gelenkentzündung hemmt. Diese neue Makrophage-Art trägt sowohl M1- als auch M2-Merkmale. M2-Makrophagen sind für die Dämpfung und Kontrolle von Entzündungen verantwortlich und werden oft von Tumoren genutzt, um sich der Zerstörung durch das Immunsystem zu entziehen.
\end{abstract}

\section{\) Gefahrenreize führen bei FR-} positiven Makrophagen zur Bildung des immunsuppressiven Adenosins

Ohradanova-Repic erklärt: „Aktivierte Makrophagen, die auf ihrer Oberfläche einen spezifischen Folatrezeptor beta (FR) tragen, sind schon länger in RA-Gelenken nachweisbar und wurden als die Entzündungsförderer angesehen. Aber als diese Makrophagen auch in Tumoren entdeckt wurden, vermuteten wir, dass ihre Rolle bei der rheumatoiden Arthritis eine andere, nämlich positive, sein könnte, da Tumormakrophagen entzündungshemmend wirken."

\section{Adenosin als Treiber fürs Umschal- ten von $\mathbf{M} 1$ auf $\mathbf{M} 2$ \\ Die Forscher konnten nun nachwei- sen, dass FR-positive Makrophagen auf Gefahrenstimuli wie etwa Krank-}

heitserreger in unerwarteter Weise reagieren: Sie wurden nicht proinflammatorisch, sondern wechselten in den entzündungshemmenden M2-Zustand und unterdrückten das Immunsystem, insbesondere die TZellen, stark ( $\bullet$ Abb. 1). Der dahinter liegende Mechanismus: Gefahrenreize lösen bei FR-positiven Makrophagen eine Kette von molekularen Ereignissen aus, die zur Bildung des immunsuppressiven Adenosins führt, das in der Folge die Entzündung bremst.

Darüber hinaus fanden die Wissenschafter rund um Studienleiter Prof. Dr. Hannes Stockinger von der MedUni Wien heraus, dass das herkömmliche Antirheumatikum Methotrexat die Adenosinbildung in diesen Makrophagen fördert. Um Methotrexat punktgenau zu diesen Makrophagen zu transportieren und seine Nebenwirkungen zu reduzieren, entwickelten die Forscher eine neuartige Methotrexat-Formulierung, welche die Adenosinproduktion durch die Makrophagen erhöht und die Entzündung in einem Arthritis-Mausmodell verhindern kann. Stockinger: „Die Studie betont die Kontrolle der Adenosinproduktion durch spezifische Makrophagen-Subgruppen als attraktives Ziel für therapeutische Maßnahmen bei immunvermittelten Krankheiten."

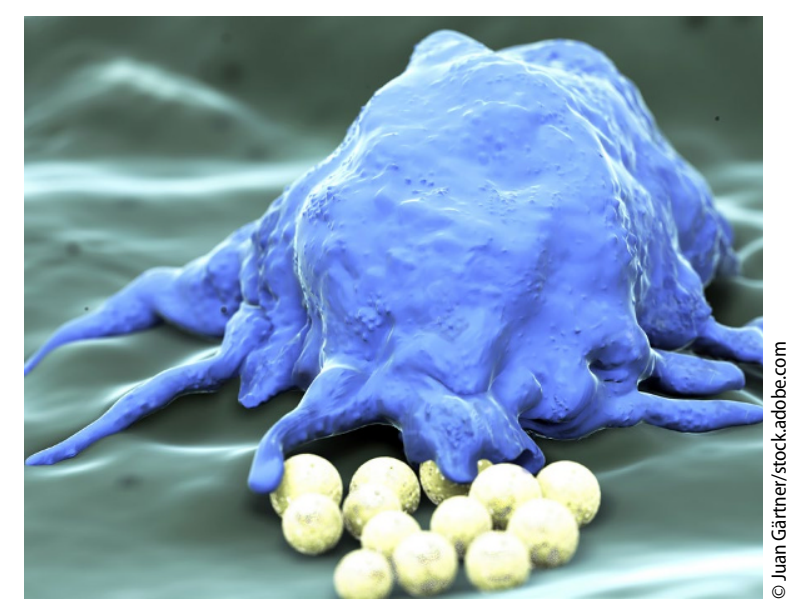

Abb. 1 \ Makrophagen können auch entzündungshemmend wirken

\section{Literatur \\ 1. Ohradanova-Repic A et al (2018) Extracellu- lar purine metabolism is the switchboard of immunosuppressive macrophages and a no- vel target to treat diseases with macrophage imbalances. Front Immunol 9:852 https:// doi.org/10.3389/fimmu.2018.00852}

rheuma plus $2018 \cdot 17: 79$

https://doi.org/10.1007/s12688-

018-0191-1

(c) Springer-Verlag GmbH Austria, ein Teil von Springer Nature 2018

Quelle: Presseaussendung MedUni Wien
Hier steht eine Anzeige.

$$
\text { 黛 Springer }
$$

